\title{
Preface to the JOT Special Issue dedicated to Martin Gogolla on his 65th Birthday
}

\author{
Lars Hamann*, Richard F. Paige ${ }^{\dagger}$, Alfonso Pierantonio ${ }^{\ddagger}$, Bernhard Rumpe $^{\S}$, and Antonio Vallecillo ${ }^{\odot}$ \\ *University of Applied Sciences Hamburg, Germany \\ ${ }^{+}$McMaster University, Canada \\ łUniversità degli Studi dell'Aquila, Italy \\ §RWTH Aachen University, Germany \\ •ITIS Software, Universidad de Málaga, Spain
}

\begin{abstract}
This issue contains a collection of essays dedicated to Prof. Dr. Martin Gogolla on his 65th birthday. The articles aim to acknowledge Martin's substantial contributions to software technology throughout his career, starting with his Ph.D. (if not already his Master thesis) through to the present day. Martin's efforts have helped modeling languages such as OCL and UML become more rigorous and precise, and have made the USE modelling tool a significant and well-known research vehicle.
\end{abstract}

KEYWORDS OCL, UML, software specifications, software modeling, program analysis, software testing.

\section{Introduction}

This special issue presents a collection of research papers about practical approaches to rigorous software modeling. These approaches feature two significant characteristics, which are generally challenging to combine. Firstly, they have solid formal foundations, something required to provide the rigor needed to build precise software models and enable reasoning about them. Secondly, these approaches are inherently practical and usually supported by useful tools to be used by software engineers and practitioners to model their systems in a practical and usable manner.

This is precisely the approach espoused throughout his career by Prof. Martin Gogolla. In grateful acknowledgment of his invaluable and fundamental activities in the field, this special issue is dedicated to him on the occasion of his retirement, which is by German law upon reaching the 65th birthday. Martin has made a substantial number of significant contributions to software technology and conceptual modeling, particularly in the contexts of UML (Object Management Group 2015) and OCL (Object Management Group 2014). Martin has dedicated all his energy

\section{JOT reference format:}

Lars Hamann, Richard F. Paige, Alfonso Pierantonio, Bernhard Rumpe, and Antonio Vallecillo. Preface to the JOT Special Issue dedicated to Martin Gogolla on his 65th Birthday. Journal of Object Technology. Vol. 19, No. 3, 2020. Licensed under Attribution 4.0 International (CC BY 4.0)

http://dx.doi.org/10.5381/jot.2020.19.3.e1 over so many years to ensure rigor and solid foundations to his ideas while at the same time providing USEful tools to enable the application of his proposals in practice (Gogolla et al. 2007). The research presented in this special issue witnesses Martin's broad interests, including, among others, software specifications, software modeling, program analysis, and software testing.

Overall, this Festschrift offers a collection of high-quality articles, presenting original research results, major case studies, and inspiring visions. They have been prepared for the event in honor of Martin, announced in October 2020 in the MODELS Conference, and then held virtually in November 2020. All contributing authors are leading experts in these areas, who have collaborated with Martin over the course of his career.

\section{Prof. Dr. Martin Gogolla}

\subsection{Bio}

Martin was born shortly after Christmas on 28 December 1954. He started his journey in research at the University of Dortmund 19 years later in 1973, where he studied Mathematics and Computer Science until 1981. There he completed his Master's degree with a thesis entitled Eine Charakterisierung von nicht-strukturierten Flussdiagrammen; even at that stage he was already dealing with modelling techniques. He continued to work in the "algspec" project until 1985 with Professor Ehrich, who moved from Dortmund to Braunschweig in 1983 and took 
Martin with him. Martin and his family are still living in lovely Braunschweig.

In his Ph.D. thesis, completed at the Braunschweig University of Technology in 1986, Martin contributed to the field of abstract data types, where he investigated algebraic specifications, especially in subsorting and error handling. These technologies are two of the foundational theories underpinning objectoriented technology, especially his favorite future research topic, logical modeling with the Object Constraint Language (OCL) and related languages.

In 1986, Martin received a permanent position as "Akademischer Rat" at the TU Braunschweig, with continuous intensive research and organizational duties. Interestingly, he worked at that time in the beautiful Gausstr. 12 building, where one of the authors of this paper later had his domicile for a while. In 1994, he received his position as Full Professor for Database Systems at the University of Bremen, from which he is now retiring.

\subsection{Publications}

Martin has produced a significant number of influential publications throughout his career. The complete list of Martin's papers (288 at the time of this writing) can be found at

- www.db.informatik.uni-bremen.de/publications and

- dblp.uni-trier.de/pers/g/Gogolla:Martin.html

Among those, one of his papers with Mark Richters obtained the "10 years most influential paper award" at the MoDELS conference in 2010.

The following is a selected list of papers by Martin Gogolla, which the authors of this preface consider contains some of the works where he developed substantial new insights for the field:

- Martin Gogolla, Klaus Drosten, Udo W. Lipeck, HansDieter Ehrich: Algebraic and operational semantics of exceptions and errors. Theoretical Computer Science 1983: 141-151

- Martin Gogolla, Uwe Hohenstein: Towards a Semantic View of an Extended Entity-Relationship Model. ACM Trans. Database Syst. 16(3): 369-416 (1991)

- Martin Gogolla, Stefan Conrad, Rudolf Herzig: Sketching Concepts and Computational Model of TROLL light. DISCO 1993: 17-32

- Mark Richters, Martin Gogolla: On Formalizing the UML Object Constraint Language OCL. ER 1998: 449-464

- Martin Gogolla: Tales of ER and RE Syntax and Semantics. Transformation Techniques in Software Engineering 2005

- Martin Gogolla, Fabian Büttner, Mark Richters: USE: A UML-based specification environment for validating UML and OCL. Sci. Comput. Program. 69(1-3): 27-34 (2007)

- Jean Bézivin, Fabian Büttner, Martin Gogolla, Frédéric Jouault, Ivan Kurtev, Arne Lindow: Model Transformations? Transformation Models! MoDELS 2006: 440-453

- Mark Richters, Martin Gogolla: Validating UML Models and OCL Constraints. «UML» 2000: 265-277

- Martin Gogolla, Antonio Vallecillo: Tractable Model Transformation Testing. ECMFA 2011: 221-235
- Martin Gogolla, Frank Hilken, Khanh-Hoang Doan: Achieving model quality through model validation, verification and exploration. Comput. Lang. Syst. Struct. 54: 474-511 (2018)

\subsection{Tooling}

Martin is very much interested in the modeling of software systems. He pushed the field forward with in-depth looks in foundational and theoretical issues around behavioral modeling languages, such as Petri nets and later on algebraic specifications. He and his colleagues developed "TROLL light" (see the above publication excerpt), a tooling infrastructure that applied algebraic specification techniques to object-oriented technologies. From there, it was only a small step to the "USE" tool infrastructure that used the de facto standard OCL notation and part of the UML language for the logical specification of the structure, behavior, and properties of systems.

While USE started as a tool to visualize, simulate, and validate logical properties of textual models, it now supports a full set of modeling and analysis features. It provides a profound basis for other modeling related tools. Maybe the most significant property of USE was and still is its pragmatic and ready-touse approach. In other words, it can be used out-of-the-box and without any significant complex installation or deployment process.

Notable parts of Martin's research reside in USE. The logical specification of properties and behavior is located at the heart of USE. Around this core functionality, different approaches to validate and to verify system properties exist. Martin steadily directed the development of USE to more advanced model finding and model checking techniques. For example, the early versions of USE included a domain-specific language to search for valid model instances in a procedural style, i.e., a user-guided search.

Next, an approach requiring less guidance based on SATsolvers was added to USE. One of the last steps was to apply this model validator to sequences of method invocations by constructing special types of models called filmstrip models. This is only one example of a concrete development path in USE taken under Martin's supervision.

Furthermore, the simplicity and stability of USE might be reasons why it is widely used for teaching modeling concepts and techniques in many universities beyond Bremen. The fact that it is continuously downloaded more than 100 times a week over the last few years clearly shows that the USE tool has many users (and fans) in the modeling community.

He also connected these constructs of behavioral descriptions and logical restrictions to the world of database systems and especially to their entity-relationship models, which are the standard for the database community.

\subsection{Community service}

Martin has also been very active in helping the community organize itself. In 2001, only two years after its creation as an international conference series, he organized the MODELS conference. It was still called the «UML» - The Unified Modeling Language: Modeling Languages, Concepts, and Tools. In the 


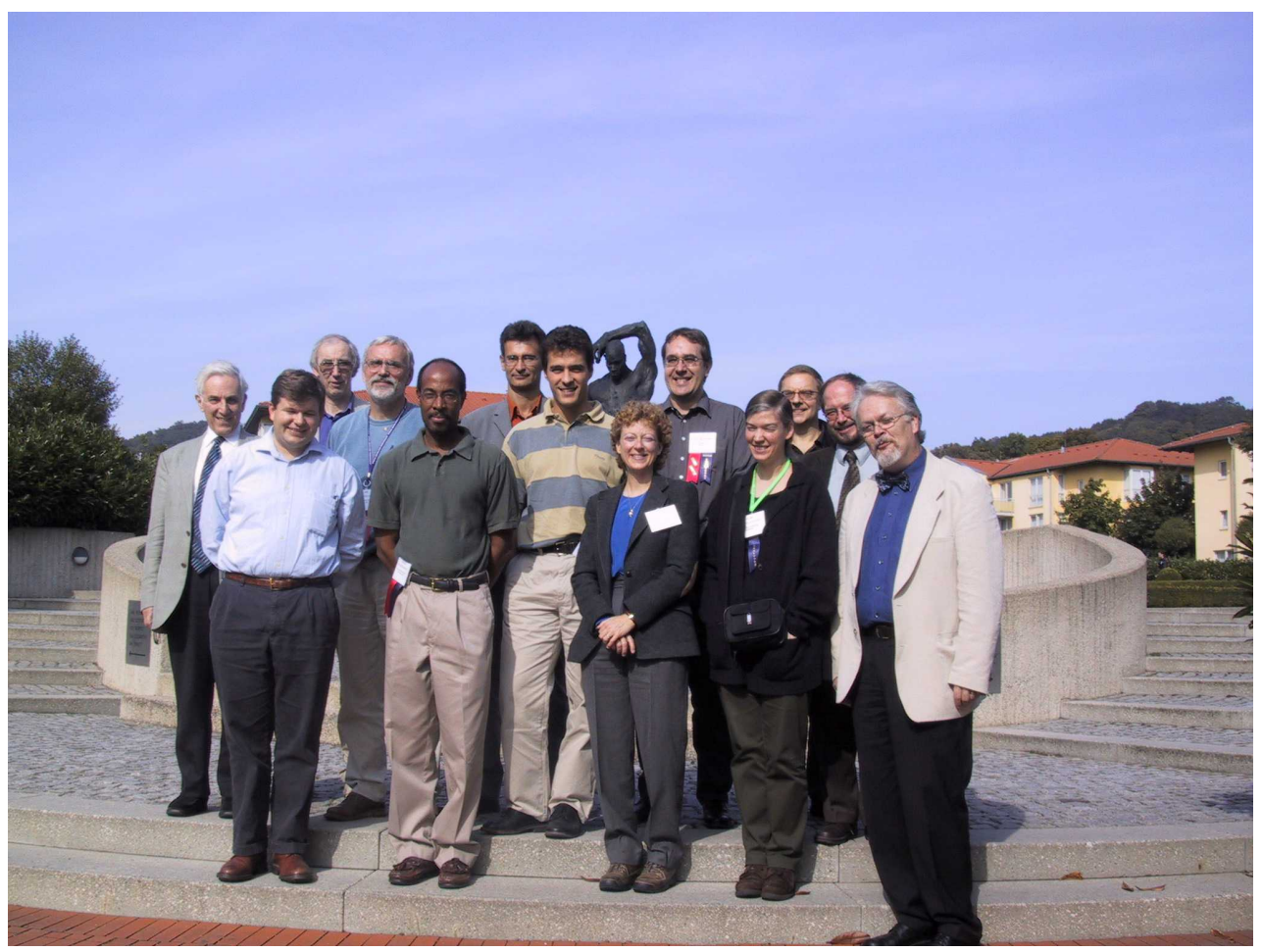

Figure 1 Group photo of the Editor Board on the occasion of the publication of the first SoSym issue in October 2002. From left to right: Michael Jackson, Steve Cook, Stuart Kent, Bran Selic, Robert France, Jean-Marc Jezequel, Jean-Michel Bruel, Geri Georg, Bernhard Rumpe, Perdita Stevens, Martin Gogolla, Heinrich Hussmann, and Hermann Engesser.

following years, Martin was very active in the steering committee of MODELS. He also became a founding editor of the Springer Journal on Software and Systems Modelling (SoSyM), created shortly after the MODELS conference series, and since 2014 is Associate Editor of the SoSyM Editor Board.

He was also one of the founding organizers of the OCL workshop series, which has been successfully running for almost 20 years with editions in the most influential modeling conferences.

Martin has been deeply engaged in other aspects of community service, for example, as a $\mathrm{PhD}$ external examiner. He is known for being an excellent examiner: rigorous, fair, and above all friendly. Students with Martin as external examiner know that their work has been judged appropriately and rigorously.

\subsection{Personal}

We should briefly mention a few personal aspects of Martin's life. He is very family oriented, and talks often of his wife Brigitte, his children, and his cat. When at conferences, he is often up early and running first thing in the morning, putting younger members of the community to shame!

\section{Contents of this special issue}

The papers featured in this special issue survey and bring new perspectives to the fields of software specification, software modeling, program analysis, and software testing.

The organizers contacted prospective authors among those researchers who have closely worked and collaborated with Martin throughout his career. It was interesting to realize how large the number of Martin's colleagues was and how all of them appreciated his work and contributions. Unfortunately, not all contacted authors could write something for the special issue, in many cases, due to the particular circumstances of the Covid-19 situation. In all cases, though, the response was overwhelmingly appreciative and supportive.

In the end, we received 22 tentative proposals, of which 20 became full submissions by the final deadline. Being a journal publication, all submitted papers were thoroughly peerreviewed by two other reviewers for adequacy, novelty, rigor, and technical quality.

The final revised papers comprise this special issue. They are the following

- Paula Muñoz, Loli Burgueño, Victor Ortiz and Antonio Vallecillo in their paper entitled Extending OCL with Subjective Logic propose an extension of the OCL/UML datatype Boolean that enables the representation of subjective uncertain opinions for reasoning with uncertain propositions in order to reach better informed decisions.

- Bran Selic, in his paper entitled The Forgotten Interfaces: A Critique of Component-based Models of Computing and a Remedy, analyzes the nature of the relationship between software and its supporting hardware to give the notion of engineering correctness as something that is fundamental to reliable software design.

- Achim D. Brucker, Frédéric Tuong, and Burkhart Wolff, in their paper entitled Model-Transformation as Conservative Theory-Transformation, present a new technique to con- 
struct tool support for domain-specific languages (DSLs) with higher-order logic (HOL) inside the interactive theorem prover environment Isabelle.

- Arvid Butting, Robert Eikermann, Katrin Hölldobler, Nico Jansen, Bernhard Rumpe and Andreas Wortmann, in their paper entitled A Library of Literals, Expressions, Types, and Statements for Compositional Language Design, present an extensible library of language components to engineering new languages through their reuse. The proposal aims at providing more efficient language engineering features in MontiCore.

- Pablo Gómez-Abajo, Esther Guerra, Juan De Lara, and Mercedes G. Merayo, in their paper entitled Systematic Engineering of Mutation Operators, propose a methodology and corresponding tool support for engineering mutation operators. A language for mutation operators facilitates the synthesis of models that can be used to test the operators, different metrics to analyse operator coverage, and services to generate operators when the coverage is insufficient.

- Fiona Polack and and Kieran Alden, in their contribution entitled Engineering dynamic systems: from behaviour to code, explore issues in the use of model transformation for simulation development, using a Java Mason simulator, created using the CoSMoS approach from UML-style state diagrams.

- Jordi Cabot, Robert Clarisó, and Carlos A. González, in their paper entitled Incremental Verification of UML/OCL models, propose techniques for the incremental verification of the internal consistency of UML class diagrams annotated with OCL constraints. Such techniques allow modelers to significantly reduce the cost of re-verifying a class diagram after model updates.

- Karsten Sohr, Christian Maeder, Rodrigue Wete Nguempnang, Nils Meyer-Larsen, and Rainer Müller, in their contribution entitled Modeling and Validating Role-Based Authorization Policies for a Port Communication System with $U M L$ and $O C L$, propose an approach to apply modeling to the field of information security. The approach is based on a combination of Role-Based Access Control (RBAC) with constraints and Attribute-Based Access Control (ABAC) for finer grained authorization constraints.

- Hans-Jörg Kreowski and Sabine Kuske, in their paper entitled Modeling and Analyzing Graph Algorithms by Means of Graph Transformation Units, present the concept of graph transformation units as a framework to model graph algorithms, to prove their correctness and to analyze their complexity with a specific focus on the improvement of the efficiency by massive parallelism.

- Alexander Knapp and María Victoria Cengarle, in their paper entitled $O C L$ as a 2-term charter, motivated by the OCL and its contract language, present a systematic transition from term charter domains and term charters for evaluating general expressions over a single system state, to 2-term charter domains and 2-term charters involving two system states.

- Tony Clark, in his paper A Meta-Circular Basis for ModelBased Language Engineering, provides a step-by-step boot- strap of a meta-circular language for language engineering alongside with a collection of key characteristic features that are used to define its foundations.

- Kristóf Marussy, Oszkár Semeráth, Aren Babikian, and Daniel Varro, in their contribution entitled A specification language for consistent model generation based on partial models, present a specification language for partial models used in consistent graph model generation, whose semantics is defined by using 4-valued Belnap-Dunn logic that explicitly marks inconsistent model elements as part of the partial model.

- Christoph Bockisch, Gabriele Taentzer, Nebras Nassar, and Lukas Wydra, in their paper entitled Java Bytecode Verification with OCL: Why, How and When?, propose to perform bytecode manipulation by using concepts and techniques of model-driven engineering. In particular, the declarative nature of model transformation rules allows debugging and analyzing bytecode manipulations in more detail than classically done.

- Alexandra Mazak, Sabine Wolny, Abel Gomez, Jordi Cabot, Manuel Wimmer, and Gerti Kappel, in their paper entitled Temporal Models on Time Series Databases, present a profile to annotate metamodels to automatically generate wrappers to time series databases that enable storing model updates as well as querying historical model information.

- Hoàng Nguyen Phuoc Bao and Manuel Clavel, in their paper entitled Model-based characterization of fine-grained access control authorization for $S Q L$ queries, propose a model-based characterization of fine-grained access control (FGAC) authorization for SQL queries using a SecureUML variant and OCL.

- Azzam Maraee, Eliran Nachmani and Arnon Sturm, in their paper entitled Constraints Specification Via Tool Support: A Controlled Experiment, present a controlled experiment using USE and a Java framework to compare the effectiveness of developing model-based constraints with respect to quality, time, and confidence.

- Edward Willink, in his paper entitled Reflections on $O C L$ 2.0, shows how the well-intentioned upgrade of the simple but useful OCL 1 specification went astray as part of the UML 2 activities. The paper also discusses why OCL tool implementers treated the OCL 2 specification with unwarranted reverence and struggled to implement it as faithfully as possible.

- Jesús Sánchez Cuadrado, in his paper entitled Towards interactive, test-driven development of model transformations, explores an approach for test-driven development of model transformations based on creating test cases using a model finder in an interactive manner. The synthesis of the input models of the test cases is driven by the results of static analysis with respect to the coverage of the transformation, with the goal of instantiating input models which are not yet handled by the transformation

- Mira Balaban, Igal Khitron and Michael Kifer, in their contribution entitled Logic-based Software Modeling with FOML, describe a framework for object modeling with 
logic, its realization in a modeling tool, prove the correctness of class modeling in FOML, illustrate the process of software modeling with the tool, and present the main features of the system.

- Finally, Frank Hilken and Lars Hamann, in their paper entitled History of the USE Tool: 20 Years of UML/OCL Modeling Made in Germany, describe the principal milestones in the USE tool life cycle and its successful application in industry and academy.

\section{Final words}

For us, it has been an honor and a pleasure to organize this special issue dedicated to Prof. Dr. Martin Gogolla on the occasion of his retirement. We sincerely wish Martin a very happy retirement in Braunschweig with his family and friends. We hope he is now able to dedicate more time to his hobbies in such a lovely city. Nevertheless, we also hope that his retirement will not be a watershed moment in his life, and he will continue giving his valuable contributions to the modeling community. His vision on both sides of software modeling, the theoretical level on the rigorous specification and analysis of software systems, the applicative level with his USE tool, are irreplaceable.

\section{Acknowledgments}

We want to thank all the authors for accepting our invitation to contribute to this special issue with very interesting and highquality papers; without them, this compilation of essays and works dedicated to Martin would not have been possible. We would also like to acknowledge the JOT journal's excellent support during the preparations of the special issue.

\section{References}

Gogolla, M., Büttner, F., \& Richters, M. (2007). USE: A UML-based specification environment for validating UML and OCL. Sci. Comput. Program., 69(1-3), 27-34. doi: 10.1016/j.scico.2007.01.013

Object Management Group. (2014). Object Constraint Language (OCL) Specification. Version 2.4. (OMG Document formal/2014-02-03)

Object Management Group. (2015). Unified Modeling Language (UML) Specification. Version 2.5. (OMG document formal/2015-03-01)

\section{About the authors}

Lars Hamann is a full professor at the University of Applied Sciences Hamburg (HAW Hamburg). He is a former member of the database systems group at the University Bremen let by Martin Gogolla. His research interests include modelling and software quality. You can contact the author at lars.hamann@ hawhamburg.de.
Richard Paige is a full Professor at McMaster University, where he is director of the Centre for Software Certification. His research interests are in Model-Based Systems Engineering, certification, safety, data analytics, and formal methods. You can contact the author at paigeri@mcmaster.ca or visit https:// www.eng.mcmaster.ca/cas/people/faculty/richard-paige.

Alfonso Pierantonio is full Professor at the Università degli Studi dell' Aquila (Italy) and Editor-in-Chief of the Journal of Object Technology. His research interests include Language and Model-Driven Engineering, model management and modeling environment definition. You can contact the author at alfonso.pierantonio@univaq.it or visit http://pieranton.io.

Bernhard Rumpe is full Professor at RWTH Aachen University, where he leads the Software Engineering Research Group. His research interests include modelling, model languages, software model language engineering, code synthesis from models and model-based analysis. You can contact the author at rumpe@serwth.de or visit https://www.se-rwth.de.

Antonio Vallecillo is full Professor at the University of Málaga, where he leads the Atenea Research Group on Software and Systems Modeling. His main research interests include Open Distributed Processing, Model-based Engineering and Software Quality. You can contact the author at av@1cc.uma.es or visit http://www.lcc.uma.es/ av/. 